\title{
Author Correction: Sex-specific association between soluble corin and metabolic syndrome in Chinese adults
}

\author{
Hongmei Li · Qiu Zhang • Yan He · Jijun Shi · Weidong Hu • Hao Peng
}

Published online: 13 April 2021

(C) The Japanese Society of Hypertension 2021

Correction to: Hypertension Research

https://doi.org/10.1038/s41440-019-0228-4

published online 14 February 2019

In the version of this article originally published, the grant number associated with the Natural Science Foundation of
Jiangsu Province contained an error. In the section of Funding, the corrected grant number should be BK20180841.

The original article has been corrected. 